\title{
Por qué debemos fomentar el uso de los repositorios para compartir publicaciones y resultados de investigación en acceso abierto
}

\author{
Alicia Fátima Gómez Sánchez | Fundación Española para la Ciencia y Tecnología (FECYT) \\ URL de la contribución <www.iaph.es/revistaph/index.php/revistaph/article/view/4673>
}

Las dos vías principales para el acceso abierto son bien a través de revistas de o con opción de acceso abierto (vías dorada o diamante, en función de quién corre con los gastos de la publicación), o a través del depósito en repositorios institucionales, temáticos o generales (vía verde). Asimismo, los mandatos de organismos financiadores, las normativas legales (nacionales, regionales, institucionales) y los propios editores de libros o revistas de acceso por suscripción dejan claro que los textos completos de artículos pueden ser compartidos al cabo de embargos establecidos y en determinadas versiones (por lo general, manuscritos aceptados o postprints y manuscritos enviados o preprints) pueden ser compartidos en repositorios, pero no en otros recursos como ResearchGate (red que a menudo se cita de forma explícita) ${ }^{1}$.

A pesar de ello, numerosos estudios muestran que los investigadores anteponen el uso de redes sociales académicas (en adelante, RSA) frente al de los repositorios. A modo de ejemplo, Borrego (2017) señala que en los primeros meses de 2016 tan solo un $11 \%$ de los artículos publicados durante 2014 por investigadores afiliados a universidades españolas estaba disponible en sus repositorios institucionales; mientras que más de la mitad de los artículos publicados por esos investigadores estaba a texto completo en ResearchGate.

Un repositorio es una "infraestructura y el servicio que permiten el almacenamiento persistente, eficiente y sostenible de objetos digitales (como documentos, datos y códigos)" (BEZJAK, 2019). Además de que los repositorios ejercen una función de preservación de la información científica, que no aseguran las RSA, son depósitos reconocidos legalmente por las editoriales y por los organismos financiadores para archivar y difundir los resultados de investigación; considerando no solo las publicaciones revisadas por pares, sino también la literatura gris, los datos de investigación, etc. Entonces, ¿por qué cuando se habla de compartir información en acceso abierto redes sociales académicas como ResearchGate y Academia.edu aparecen como una alternativa, incluso preferente?

Una de las principales razones que defienden los investigadores a favor del uso de las RSA es, por ejemplo, la facilidad de agregar documentos a golpe de botón para añadir artículos o la de otorgar DOI a publicaciones $^{2}$. Sin embargo, frecuentemente desconocen los riesgos de vulnerar las políticas de copyright de revistas y editoriales al compartir trabajos en estas redes, que por supuesto hacen responsable al autor del contenido de cualquier infracción ${ }^{3}$. Los repositorios, en cambio, son reconocidos como espacios legales tanto por las editoriales como por los mandatos de los organismos financiadores. Muchos además también facilitan identificadores persistentes (DOI o handle). Asimismo los repositorios institucionales disponen de equipos que apoyan el archivo delegado o que aseguran procesos de revisión en el archivo, de forma que se minimizan los riesgos de infringir políticas editoriales de copyright.

El suministro de métricas de las redes sociales académicas (visitas a los perfiles, descargas, citas, recomendaciones, etc.) es otro de los atractivos para los investigadores. A pesar de ser algo desconocido para muchos usuarios, los repositorios también ofrecen módulos de estadísticas de descargas y visualización de documentos, enlazan con métricas alternativas y, en ocasiones, con citas de proveedores externos. Estos 


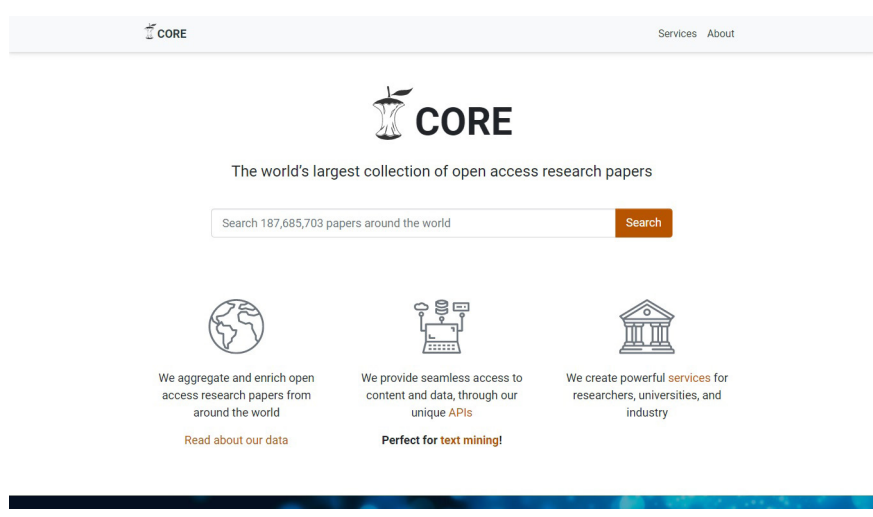

Página de inicio de Core

datos permiten a los usuarios de los repositorios analizar la repercusión académica y en redes sociales de sus publicaciones.

Pero, por encima de esto, el mayor valor añadido de los repositorios es el de la interoperabilidad. Esta se define como la capacidad para comunicar sistemas entre ellos y pasar información de ida y vuelta en un formato utilizable, que es lo que hace posible que los motores de búsqueda puedan recuperar los artículos y que los usuarios puedan descubrir información que, de otra manera, no habrían localizado (RODRIGUES; CLOBRIDGE, 2011). Prácticamente la totalidad de los repositorios cumplen al menos un estandar de metadatos como Dublin Core (DC-Metadata Element Set) y protocolos de interoperabilidad de metadatos como el Protocolo para la Recolección de Metadatos OAI$\mathrm{PMH}$ (Open Archive Initiative-Protocol for Metadata Harvesting), lo que les permite ser recolectados y ser agregados a estructuras mayores. Algunas de las plataformas o agregadores son Recolecta ${ }^{4}$, OpenAIRE ${ }^{5}$, CORE 6 o Google Scholar.

Por otra parte, la evolución de la tecnología ha hecho posible que se dé paso a la interoperabilidad semántica, asegurando que dicho intercambio tenga lugar también a nivel de contenido, lo que permite a los usuarios encontrar datos de múltiples repositorios, aumentando la relevancia en la recuperación global de información (RODRIGUES; CLOBRIDGE, 2011).

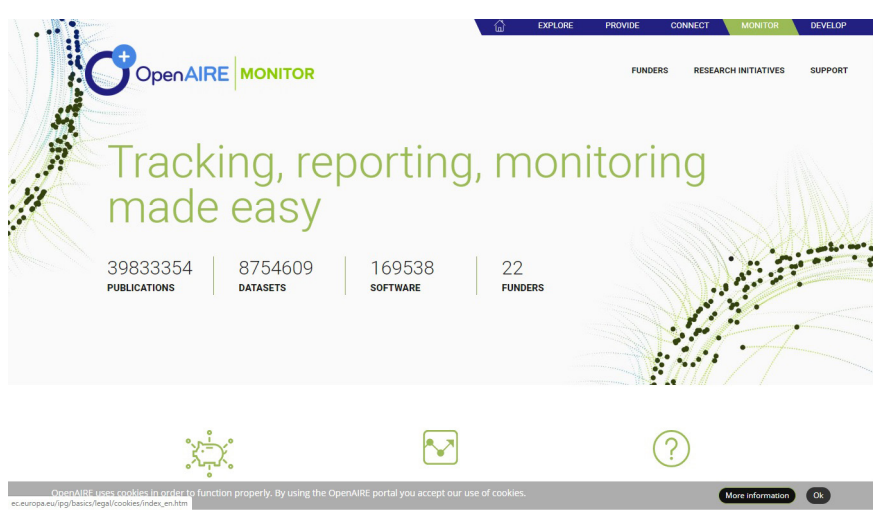

OpenAIRE Research Graph

En definitiva, queda claro que los repositorios son las estructuras más adecuadas para compartir información en acceso abierto, de manera que es imprescindible asegurar que los investigadores conozcan sus características, sus funcionalidades y sus ventajas.

Para incrementar el uso de los repositorios institucionales se debería aumentar la formación a los usuarios:

$>$ Recalcando el valor de repositorios, en especial de los institucionales, como espacio público para proporcionar acceso a la producción científica, frente a las RSA con ánimo de lucro. Esto es más significativo cuando se trata de investigación financiada con fondos públicos.

$>$ Demostrando que la interoperabilidad de los repositorios realmente aumenta la visibilidad y con ello la citabilidad de los documentos (publicaciones, sets de datos, etc.) en ellos depositados, al ser indexados por otros recursos de mayor alcance como OpenAIRE o Google Scholar.

$>$ Asegurando que los investigadores conocen las opciones legales para cumplir con las políticas de copyright de editor, así como otras funcionalidades como las estadísticas de uso y métricas que ofrecen.

$>$ Destacando el papel de las bibliotecas, que gestionan los repositorios y prestan apoyo a sus investigadores para que depositen sus trabajos a través de ayuda en las prácticas de autoarchivo o proporcionando servicios de archivo delegado. 
a debate Repositorios y redes sociales académicas para la transferencia del conocimiento abierto

| coordina Remedios Melero Melero

En conclusión, si bien las RSA son recursos diseñados en un principio para conectar e interactuar con otros colegas, las funcionalidades desarrolladas para intercambiar y almacenar información han dado paso a que se usen sobre todo como plataformas opcionales a los repositorios para compartir resultados de investigación. Sin detrimento del uso de plataformas como Researchgate o Academia.edu para aumentar la visibilidad, es evidente que los repositorios no deberían presentarse como una alternativa, sino como el lugar por defecto para almacenar y difundir cualquier resultado de investigación en acceso abierto. Si los usuarios no son conscientes de sus características y ventajas, deberemos trabajar en superar esa barrera, dando a conocer el valor añadido de los repositorios, y así hacer que se aprovechen debidamente.

\section{NOTAS}

1. ResearchGate se define como una red profesional para científicos e investigadores en la que miembros de todo el mundo comparten, descubren y debaten investigaciones, y expresa que su misión es conectar el mundo de la ciencia y hacer que la investigación esté abierta a todos. ResearchGate <https://www.researchgate.net/ about> [Consulta: 08/05/2020]. Por su parte Academia. edu expresa que es una plataforma para que los académicos compartan trabajos de investigación y que su misión de la compañía es acelerar la investigación mundial. Academia.edu <https://www.academia.edu/about $>$ [Consulta: 08/05/2020].

2. ResearchGate (2020) ResearchGate DOls <https:// explore.researchgate.net/display/support/ResearchGate+DOIs> [Consulta: 08/05/2020].

3. ResearchGate (2020) Copyright and ResearchGate $<$ https://explore.researchgate.net/display/support/Copyright> [Consulta: 08/05/2020].

4. RECOLECTA <https://www.recolecta.fecyt.es/> [Consulta: 07/05/2020].
5. OpenAIRE Research Graph <https://explore.openaire.eu/> [Consulta: 07/05/2020].

6. CORE <https://core.ac.uk/> [Consulta: 07/05/2020].

\section{BIBLIOGRAFÍA}

- BORREGO, A. (2017) Institutional repositories versus ResearchGate: The depositing habits of Spanish researchers. Learned Publishing, vol. 30, n. ${ }^{\circ}$ 3, 2017, pp. 185-192 <https:// doi.org/10.1002/leap.1099> [Consulta: 07/05/2020]

- BEZJAK, S.; CLYBURNE-SHERIN, A.; CONZETT, P. et ál. (2019) Manual de Capacitación sobre Ciencia Abierta. ed. español. sl: Foster, 2019 <https://book.fosteropenscience.eu/ es/> [Consulta: 07/05/2020]

- RODRIgues, E.; ClOBRIDGE, A. (2011) El caso de Interoperabilidad para Repositorios de Acceso Abierto. Julio 2011, versión 1.0. sl: Confederation of Open Access Repositories, 2011. <http://doi.org/10.5281/zenodo.12563> [Consulta: 07/05/2020] 